Series A

\author{
I. MATHEMATICA
}

338

\title{
ON BASIC GROUPS FOR THE SET OF FUNCTIONS OVER A FINITE DOMAIN
}

BY

ARTO SALOMAA

H E L S I N K I 1963

S U O M A L A I N E TIEDEAKATEM I A

https://doi.org/10.5186/aasfm.1964.338 
Communicated 13 September 1963 by P. J. Myrberg and K. InkerI 


\section{On basic groups for the set of functions over a finite domain}

1. Results. Let $\mathfrak{E}_{n}$ be the set of functions whose variables, finite in number, range over a fixed finite set

$$
N=\{1,2, \ldots, n\}, n \geqq 2
$$

and whose values are elements of $N$. If $\mathfrak{F} \subset \mathfrak{F}_{n}$ we denote by $\overline{\widetilde{F}}$ the closure of $\mathfrak{F}$ under composition. $\mathfrak{F}$ is said to be complete if $\overline{\mathfrak{F}}=\left(\mathfrak{E}_{n}{ }^{1}\right)$

Every complete set contains a function satisfying Slupecki conditions, i.e. depending essentially on at least two variables and assuming all $n$ values. We say that a subset $\mathfrak{F}$ of $\mathfrak{E}_{n}$ is a basic set for $\mathfrak{E}_{n}$ if $\mathfrak{F}$ is not complete but the addition to $\mathfrak{F}$ of any function satisfying Słupecki conditions yields a complete set. If a basic set is a group with respect to composition it is termed a basic group for $\mathfrak{E}_{n}$.

It is shown in [1, pp. 72-76] that all 1-place functions belonging to $\mathfrak{F}_{n}$ form a basic set $\widetilde{F}_{1}$ for $\mathfrak{E}_{n}$, provided $n \geqq 3$. This result has been strengthened to concern various subsets of $\widetilde{F}_{1}$ which are closed under composition. It is shown in [1] that the subset of $\mathfrak{F}_{1}$ consisting of all 1-place functions other than permutations is a basic set for $\mathfrak{E}_{n}$, provided $n \geqq 3$.

On the other hand, it is shown in [2] that the symmetric group of degree $n$ is a basic group for $\left(\tilde{E}_{n}{ }^{2}\right)$ Furthermore, according to [3], the alternating group of degree $n$ is a basic group for $\mathfrak{E}_{n}$. (Obviously, the latter result implies the former.) These results are valid for all values of $n \geqq 5$. Counterexamples presented in [2] show that they are not valid for $n=2,3,4$.

In this paper, we shall study the problem whether it is still possible to reduce basic groups, i.e. whether the alternating group can be replaced by a smaller group of degree $n$, provided $n \geqq 5$. In proofs of completeness criteria for subsets of $\tilde{E}_{n}$, the essential fact concerning groups is the degree of transitivity. Therefore, it is natural to ask whether the alternating group can be replaced by an arbitrary group of degree $n$ with some lower limitation on the degree of transitivity.

1) For a detailed discussion, cf. [1, pp. 56-58]. Throughout this paper, $n$ means the number of elements in the set $N$. result.

$\left.{ }^{2}\right)$ In fact, a slight modification in the proof of the theorem in [2] will yield this 
It is clear that an arbitrary doubly transitive group is not basic for $\mathfrak{F}_{n}$. Counter-examples are found, for instance, by considering prime values of $n$ and linear groups. A triply transitive group is basic for $\mathfrak{E}_{n}$ if $n$ is not a power of 2 . A quadruply transitive group is always basic for $\mathfrak{E}_{n}$ (provided the condition $n \geqq 5$ is satisfied). These results are due to the following theorem which we shall prove in sestion 2 .

Theorem. Every quadruply transitive group of degree $n$ is a basic group for $\mathfrak{E}_{n}$, provided $n \geqq 5$. If, in addition, $n \neq 2^{r}$ then every triply transitive group of degree $n$ is a basic group for $\mathbb{E}_{n}$.

It is a consequence of this theorem that if a quadruply transitive group of degree $n$ is contained in the closure of a function $f \in \mathcal{E}_{n}$ (i.e. if $f$ generates a quadruply transitive group) then the unit set of $f$ is complete. ${ }^{1}$ ) The same statement holds true for triply transitive groups of degree $n$, provided $n \neq 2^{r}, r \geqq 3$. It is very likely that the statement holds true for arbitrary triply transitive groups, perhaps even for arbitrary doubly transitive groups if $n \geqq 3$.

In section 3, we consider the exceptional cases in our theorem: $n=2^{r}$, $r \geqq 3$. We construct a triply transitive group of degree $2^{r}$ which is not a basic group for $\mathfrak{F}_{2^{r}}$. Such a counter-example is provided by the holomorph of an Abelian group of order $2^{r}$ and type $(1,1, \ldots, 1)$.

2. Proofs. To prove our theorem, we shall first establish several lemmas. We shall use the terms genus and type (of 1-place functions belonging to $\mathfrak{F}_{n}$ ) as defined in [3]. Assume that $G_{i}, i=1, \ldots, k$, are non-empty subsets of $N$. Then, for any function $f\left(x_{1}, \ldots, x_{k}\right) \in \mathcal{E}_{n}$, we denote by $f\left(G_{1}, \ldots, G_{k}\right)$ the set of values assumed by $f$ when, for $i=1, \ldots, k$, the variable $x_{i}$ is restricted to the set $G_{i}$.

Lemmas 1 and 2 are the same as lemmas 1.1 and 1.3 in [3]. Therefore, we omit their proofs.

Lemma 1. Assume that $n \geqq 3$ and $f\left(x_{1}, \ldots, x_{k}\right)$ satisfies Stupecki condiiions. Then for any $j, 3 \leqq j \leqq n$, there are sets $G_{i}, i=1, \ldots, k$, each consisting of a most $j-1$ elements such that $f\left(G_{1}, \ldots, G_{k}\right)$ contains at least $j$ elements.

Lemma 2. The set of functions of type $\left[b_{1}, b_{2}, b_{3}, \ldots, b_{t}\right]$ where $1<t<n$ generates every function of type $\left[b_{1}+b_{2}, b_{3}, \ldots, b_{t}\right]$.

Lemma 3. Assume that $n \geqq 4$ and $\widetilde{F} \subset \mathbb{E}_{n}$ contains a triply transitive group (S) (of degree $n$ ) and a function $f\left(x_{1}, \ldots, x_{k}\right)$ satisfying Slupecki conditions. Then $\mathfrak{F}$ generates a function of genus 2 and all functions of genus 1 .

\footnotetext{
1) This means that $f$ is a so-called Sheffer function. The result is valid for $n \geqq 4$ because, according to [3], it is valid for $n=4$.
} 
Proof. I. We shall first prove that $\mathfrak{F}$ generates a function $g(x)$ whose genus $\gamma$ satisfies $1<\gamma<n$.

By lemma 1, there are numbers $a_{1}, \ldots, a_{k}$ such that

$$
f\left(G_{1}, \ldots, G_{k}\right)=N
$$

where $G_{i}=N-\left\{a_{i}\right\}$, for $i=1, \ldots, k$. By (1), there are numbers $a_{i}^{\prime}, i=1, \ldots, k$, such that

$$
f\left(a_{1}^{\prime}, \ldots, a_{k}^{\prime}\right)=f\left(a_{1}, \ldots, a_{k}\right)
$$

and $a_{i}^{\prime} \neq a_{i}$, for $i=1, \ldots, k$. We choose from (S) $k$ permutations $p_{i}(x), i=1, \ldots, k$, such that $p_{i}(1)=a_{i}$ and $p_{i}(2)=a_{i}^{\prime}$. The choice is possible because (5) is doubly transitive. Then the function

$$
f\left(p_{1}(x), \ldots, p_{k}(x)\right)
$$

is of genus smaller than $n$. If it is of genus greater than 1 we have found a function $g(x)$ as required.

We, therefore, assume that the function (2) is of genus 1. Hence, $\mathfrak{F}$ generates all functions of genus 1, i.e. all constants. Using lemma 1, we choose sets $H_{i}, i=1, \ldots, k$, such that each $H_{i}$ consists of two (not necessarily distinct) elements $b_{i}$ and $b_{i}^{\prime}$ and $f\left(H_{1}, \ldots, H_{k}\right)$ contains at least three distinct elements $b, b^{\prime}$ and $b^{\prime \prime}$. By a suitable renumbering of the variables, this choice can be made in such a way that

$$
\begin{aligned}
& f\left(b_{1}, b_{2}, \ldots, b_{k}\right)=b, \\
& f\left(b_{1}^{\prime}, b_{2}, \ldots, b_{k}\right)=b^{\prime}
\end{aligned}
$$

and

$$
f\left(b_{1}^{\prime}, b_{2}^{\prime}, \ldots, b_{k}^{\prime}\right)=b^{\prime \prime}
$$

Consider the 1-place function

$$
g_{1}(x)=f\left(x, b_{2}, \ldots, b_{k}\right)
$$

which is generated by $\mathfrak{F}$. If $g_{1}(x)$ does not assume the value $b^{\prime \prime}$ we may choose $g(x)=g_{1}(x)$. Suppose

$$
g_{1}\left(c_{1}\right)=b^{\prime \prime} .
$$

Then necessarily $c_{1} \neq b_{1}, b_{1}^{\prime}$. Choose numbers $c_{2}$ and $c_{3, i}, i=2, \ldots, k$, such that $c_{2} \neq b_{1}, b_{1}^{\prime}, c_{1}$ and $c_{3, i} \neq b_{i}, b_{i}^{\prime}$ if $b_{i} \neq b_{i}^{\prime}$ but $c_{3, i}=b_{i}$ if $b_{i}=b_{i}^{\prime}$. The choice is possible because $n \geqq 4$.

Assume that

$$
f\left(c_{2}, c_{3,2}, \ldots, c_{3, k}\right)=b^{\prime \prime}
$$


Let $q_{i}(x), i=1, \ldots, k$, be constants in $\overline{\mathfrak{F}}$ or permutations in $\mathscr{B S}$, defined as follows. The function $q_{1}(x)$ is a permutation such that $q_{1}(1)=c_{2}$, $q_{1}(2)=b_{1}$ and $q_{1}(3)=b_{1}^{\prime}$. Let $2 \leqq i \leqq k$ and $b_{i} \neq b_{i}^{\prime}$. Then $q_{i}(x)$ is a permutation such that $q_{i}(1)=c_{3, i}, q_{i}(2)=b_{i}$ and $q_{i}(3)=b_{i}^{\prime}$. Let $2 \leqq i \leqq k$ and $b_{i}=b_{i}^{\prime}$. Then $q_{i}(x)=b_{i}$. By (3), (5) and (7), we may choose

$$
g(x)=f\left(q_{1}(x), \ldots, q_{k}(x)\right)
$$

Assume that

$$
f\left(c_{2}, c_{3,2}, \ldots, c_{3, k}\right)=b^{\prime \prime} .
$$

Let $q_{1}^{\prime}(x)$ be a permutation in (5) such that $q_{1}^{\prime}(1)=c_{2}, q_{1}^{\prime}(2)=c_{1}$ and $q_{1}^{\prime}(3)=b_{1}^{\prime}$. By (5), (6) and (8), we may choose

$$
g(x)=f\left(q_{1}^{\prime}(x), q_{2}(x), \ldots, q_{k}(x)\right) .
$$

Thus, in all cases, $\tilde{F}$ generates a function $g(x)$ whose genus $\gamma$ satisfies $1<\gamma<n$.

II. Assume that $\gamma>2$. We shall now prove that $\mathfrak{F}$ generates a function $h(x)$ whose genus $\gamma_{1}$ sa isfies $2 \leqq \gamma_{1}<\gamma$. By repeating the argument, we may conclude that $\mathfrak{F}$ generates a function of genus 2 .

Let $u$ be a value assumed by $g(x)$ at least twice and let $v$ and $w$ be any other distinct values of $g(x)$. Hence, there are distinct numbers $u_{1}$, $u_{2}$ and $v_{1}$ such that

$$
g\left(u_{1}\right)=g\left(u_{2}\right)=u \text { and } g\left(v_{1}\right)=v .
$$

Choose from (55 a permutation $p(x)$ such that $p(u)=u_{1}, p(u)=u_{2}$ and $p(v)=v_{1}$. Then the function

$$
h(x)=g p g(x)
$$

is of genus $\gamma_{1}$ where $2 \leqq \gamma_{1}<\gamma$.

We have, thus, shown that $\tilde{f}$ generates a function $h_{1}(x)$ of genus 2 . Let $h_{1}\left(d_{1}\right)=h_{1}\left(d_{2}\right)=d, d_{1} \neq d_{2}$, and $h_{1}\left(d_{3}\right)=d^{\prime}, d^{\prime} \neq d$. To complete the proof of lemma 3 , we choose from (5) a permutation $q(x)$ such that $q(d)=d_{1}$ and $q\left(d^{\prime}\right)=d_{2}$. Then $h_{1} q h_{1}(x)=d$. Thus, $\tilde{F}$ generates the constant $d$. Because $\mathfrak{F}$ contains a transitive group, we may conclude that $\mathfrak{F}$ generates all constants. Hence, lemma 3 follows.

Lemma 4. Assume that $n \geqq 3^{1}$ ) and $\widetilde{F} \subset \mathbb{E}_{n}$ contains a triply transitive group (S) (of degree $n$ ), a function $f\left(x_{1}, \ldots, x_{k}\right)$ satisfying Stupecki conditions and a function $g(x)$ of type $[n-1,1]$. Then $\mathfrak{F}$ is complete.

1) For the proof of our theorem, it obviously suffices to consider the cases $n>4$. A sharper formulation is given to some of the lemmas because their proofs remain unaltered. On the other hand, lemmas 4 and 5 may be considered as completeness criteria for subsets of $\mathfrak{E}_{n}, n \geqq 3$. 
Proof. Obviously, any function of type $[n-1,1]$ may be expressed in the form $p_{1} g p_{2}(x)$ where $p_{1}(x)$ and $p_{2}(x)$ are permutations belonging to $(5)$. In fact, $p_{2}$ may be chosen from any transitive subgroup of $\mathbb{G S}$ and $p_{1}$ may be chosen from any doubly transitive subgroup of $\mathfrak{S}$. Thus, $\mathfrak{F}$ generates all functions of type $[n-1,1]$.

We shall now make the following hypothesis of induction: $\mathfrak{F}$ generates all functions of type

$$
[n-i, \underbrace{1, \ldots, 1]}_{i \text { terms }}
$$

where $1 \leqq i<n-2$. We shall prove that this implies that $\mathfrak{F}$ generates all functions of type

$$
[n-(i+1), \underbrace{1, \ldots, 1]}_{i+1} .
$$

We choose numbers $b_{i}$ and $b_{i}^{\prime}, i=1, \ldots, k$, as in the proof of lemma 3 such that the equations $(3)-(5)$ hold, for some distinct numbers $b, b^{\prime}$ and $b^{\prime \prime}$.

Let $h(x)$ be an arbitrary function of type (10). We have to show that $h(x) \in \widetilde{\mathfrak{F}}$.

The function $h(x)$ assumes $i+2$ distinct values. Let $\alpha_{1}$ be the value assumed by $h(x) n-(i+1)$ times and let $U$ consist of all numbers $y$ such that $h(y)=\alpha_{1}$. Hence, the cardinality of $U$ (denoted by $\left.\operatorname{card}(U)\right)$ is at least 2 . Finally, let the other values assumed by $h(x)$ be $\alpha_{2}, \ldots, \alpha_{i+2}$ and let $u_{v}$ be numbers such that $h\left(u_{v}\right)=\alpha_{v}$, for $v=2, \ldots, i+2$.

We choose from (5) a permutation $p(x)$ such that $p\left(b^{\prime}\right)=\alpha_{1}, p(b)=\alpha_{2}$ and $p\left(b^{\prime \prime}\right)=\alpha_{3}$ and denote

$$
f_{1}\left(x_{1}, \ldots, x_{k}\right)=p\left(f\left(x_{1}, \ldots, x_{k}\right)\right)
$$

Clearly, $f_{1}\left(x_{1}, \ldots, x_{k}\right)$ satisfies Słupecki conditions. Therefore, it is possible to choose numbers $\alpha_{v}^{\mu}, \mu=1, \ldots, i-1, v=1, \ldots, k$, such that $f_{1}$ applied to the $\mu^{\text {th }}$ row vector of the matrix

$$
\| \begin{array}{ccc}
\alpha_{1}^{1} & \ldots & \alpha_{k}^{1} \\
\vdots & \vdots \\
\alpha_{1}^{i-1} & \ldots & \alpha_{k}^{i-1}
\end{array} \mid
$$

yields the value $\alpha_{\mu+3}$, for any $\mu=1, \ldots, i-1$.

We now consider auxiliary functions $h_{i}(x), i=1, \ldots, k$, defined by the following table: 


\begin{tabular}{|l|c|c|c|c|}
\hline & $h_{1}(x)$ & $h_{2}(x)$ & $\ldots$ & $h_{k}(x)$ \\
\hline & & & & \\
$x \in U$ & $b_{1}^{\prime}$ & $b_{2}$ & & $b_{k}$ \\
$x=u_{2}$ & $b_{1}$ & $b_{2}$ & & $b_{k}$ \\
$x=u_{3}$ & $b_{1}^{\prime}$ & $b_{2}^{\prime}$ & & $b_{k}^{\prime}$ \\
$x=u_{4}$ & $\alpha_{1}^{1}$ & $\alpha_{2}$ & & $\alpha_{k}^{1}$ \\
. & & & & \\
. & & & & \\
. & & & & \\
$x=u_{i+2}$ & $\alpha_{1}^{i-1}$ & $\alpha_{2}^{i-1}$ & & $\alpha_{k}^{i-1}$ \\
\hline
\end{tabular}

It follows from our inductive assumption concerning functions of type (9) and lemma 2 that every function assuming some value at least $n-i$ times is generated by $\widetilde{F}$. Because the functions $h_{i}(x)$ satisfy this condition, we may conclude that $h_{i}(x) \in \overline{\widetilde{F}}$, for $i=1, \ldots, k$.

It is a consequence of (11) and the choice of the functions $h_{i}(x)$ that

$$
h(x)=f_{1}\left(h_{1}(x), \ldots, h_{k}(x)\right) .
$$

Thus, we have shown that all functions of type (10) are generated by $\widetilde{F}$.

We conclude, by induction, that all functions of type

$$
[2, \underbrace{1, \ldots, 1}_{n-2}]
$$

are generated by $\mathfrak{F}$. By lemma 2, the set of functions of type (12) generates the subset of $\mathfrak{E}_{n}$ consisting of all 1-place functions other than permutations. By the criterion mentioned in section 1 , we may conclude that $\mathfrak{F}$ is complete.

Lemma 5. Assume that $n \geqq 3$ and $\mathfrak{F} \subset \mathfrak{E}_{n}$ contains a triply transitive group $\mathbb{S}$ (of degree $n$ ), a function $f\left(x_{1}, \ldots, x_{k}\right)$ satisfying Stupecki conditions and a function $g(x)$ of type $[n-a, a]$ where $a \neq \frac{n}{2}$. Then $\mathfrak{F}$ is complete.

Proof. If $n=3$ or $n=4$ the assumptions of lemmas 4 and 5 are equivalent. Therefore, we assume that $n \geqq 5$. We shall show that $\mathfrak{F}$ generates a function of type $[n-1,1]$. This implies, by lemma 4, that $\mathfrak{F}$ is complete.

By the hypothesis, $n-a \neq a$. We assume that the notation is chosen in such a way that $n-a>a$. If $a=1$ the proof is completed. We, therefore, assume that $a \geqq 2$. We shall show that $\mathfrak{F}$ generates a function $g_{1}(x)$ of type $\left[n-a_{1}, a_{1}\right]$ where $1 \leqq a_{1}<a$. By repeating the argument, we conclude that $\mathfrak{F}$ generates a function of type $[n-1,1]$. 
Let $E_{1}$ and $E_{2}$ be disjoint subsets of $N$ such that $\operatorname{card}\left(E_{1}\right)=n-a$, $\operatorname{card}\left(E_{2}\right)=a \geqq 2$ and $g(x)$ assumes a constant value both in $E_{1}$ and in $E_{2}$. Because $\mathfrak{F}$ contains a doubly transitive group it generates every function assuming a constant value both in $E_{1}$ and in $E_{2}$.

We choose from $\mathfrak{S S}$ a permutation $p(x)$ mapping some element of $E_{2}$ into itself and some other element of $E_{2}$ into $E_{1}$. Consider the sets

$$
\begin{array}{ll}
V_{1}=E_{1} \cap p\left(E_{1}\right), & V_{2}=E_{2} \cap p\left(E_{1}\right), \\
V_{3}=E_{2} \cap p\left(E_{2}\right), & V_{4}=E_{1} \cap p\left(E_{2}\right) .
\end{array}
$$

The union of the sets (13) equals $N$. On the other hand, by the choice of the permutation $p$,

$$
1 \leqq \operatorname{card}\left(V_{i}\right)<\operatorname{card}\left(E_{2}\right)=a, \text { for } i=2,3,4 .
$$

Furthermore, $1 \leqq \operatorname{card}\left(V_{1}\right)$. The sets (13) are not of the same cardinality. For if card $\left(V_{1}\right)=\operatorname{card}\left(V_{2}\right)$ and $\operatorname{card}\left(V_{3}\right)=\operatorname{card}\left(V_{4}\right)$ we obtain

$$
\operatorname{card}\left(V_{1}\right)=\frac{1}{2} \operatorname{card}\left(E_{1}\right)>\frac{1}{2} \operatorname{card}\left(E_{2}\right)=\operatorname{card}\left(V_{3}\right) \text {. }
$$

Let $b_{i}$ and $b_{i}^{\prime}, i=1, \ldots, k$, be the same numbers as in the proof of lemma 3. Thus, equations (3) - (5) hold, for some distinct numbers $b, b^{\prime}$ and $b^{\prime \prime}$. Choose arbitrary elements $v_{i} \in V_{i}, i=1,2,3$, and a permutation $p^{\prime}(x) \in \mathbb{S} S$ such that $p^{\prime}(b)=v_{1}, p^{\prime}\left(b^{\prime}\right)=v_{2}$ and $p^{\prime}\left(b^{\prime \prime}\right)=v_{3}$.

The following auxiliary functions $h_{i}(x)$ are generated by $\mathfrak{F}$ :

$$
h_{i}\left(E_{1}\right)=\left\{b_{i}\right\}, h_{i}\left(E_{2}\right)=\left\{b_{i}^{\prime}\right\}, i=1, \ldots, k .
$$

(Some of the functions $h_{i}(x)$ may be constants which are generated by $\widetilde{F}$, according to lemma 3.) Let

$$
\bar{g}(x)=p^{\prime}\left(f\left(h_{1}(x), h_{2} p^{-1}(x), \ldots, h_{k} p^{-1}(x)\right)\right) .
$$

It follows from the definitions of the functions involved that

$$
\bar{g}(x)=v_{i}, \text { for } x \in V_{i}, \quad i=1,2,3 .
$$

Furthermore, $\bar{g}(x)$ assumes a constant value $v^{\prime}$, for $x \in V_{4}$.

Suppose $v^{\prime} \notin V_{4}$. Then $\bar{g}^{2}(x)$ is a function of genus 3 and type $\left[t_{1}, t_{2}, t_{3}\right]$ where at least one of the numbers $t$, say $t_{3}$, satisfies $1 \leqq t_{3}<a$. This is due to (14) and the fact that $v^{\prime} \in V_{1} \cup V_{2} \cup V_{3}$. Let the values assumed by $\bar{g}^{2}(x)$ be $u_{1}, u_{2}$ and $u_{3}$ where $u_{1}$ is assumed at least twice and $u_{3}$ exactly $t_{3}$ times. Choose numbers $u_{1}^{1}, u_{1}^{2}$ and $u_{3}^{1}$ such that $\bar{g}^{2}\left(u_{1}^{1}\right)=$ $\bar{g}^{2}\left(u_{1}^{2}\right)=u_{1}$ and $\bar{g}^{2}\left(u_{3}^{1}\right)=u_{3}$. Furthermore, choose a permutation $p_{1}(x) \in \mathbb{S} 5$ mapping the ordered triple $\left(u_{1}, u_{2}, u_{3}\right)$ into the ordered triple $\left(u_{1}^{1}, u_{1}^{2}, u_{3}^{1}\right)$. Then we may choose

$$
g_{1}(x)=\bar{g}^{2} p_{1} \bar{g}^{2}(x) .
$$

Clearly $g_{1}(x)$ is of type $\left[n-t_{3}, t_{3}\right]$ where $1 \leqq t_{3}<a$. 
Thus, we may assume that $v^{\prime}=v_{4} \in V_{4}$. The equations (15) may be written in the form

$$
\bar{g}(x)=v_{i}, \text { for } x \in V_{i}, \quad i=1,2,3,4 .
$$

We say that a quadruple $\left(\zeta_{1}, \zeta_{2}, \zeta_{3}, \zeta_{4}\right)$ is a permissible set of representatives for the numbers $v_{i}$ if there is a permutation in $\$ 5$ mapping $v_{i}$ into $\zeta_{i}$, $i=1,2,3,4$. Assume that the elements of some permissible set of representatives are contained in exactly three sets $V_{i}$ and let $p_{\xi}(x)$ be the corresponding permutation. Then the function $\bar{g} p_{s} \bar{g}(x)$ is of type $\left[t_{1}, t_{2}, t_{3}\right]$ where $1 \leqq t_{3}<a$. Proceeding as above, we obtain a function $g_{1}(x)$ as required. We may, therefore, assume that there is no permissible set of representatives whose elements are contained in exactly three sets $V_{i}$.

We shall now make use of the fact established above that the sets (13) are not of the same cardinality. If $\alpha(i)$ is a permutation of the numbers $1,2,3,4$ such that

$$
\operatorname{card}\left(V_{\alpha(1)}\right) \geqq \operatorname{card}\left(V_{\alpha(2)}\right) \geqq \operatorname{card}\left(V_{\alpha(3)}\right) \geqq \operatorname{card}\left(V_{\alpha(4)}\right)
$$

then necessarily

$$
\operatorname{card}\left(V_{\alpha(1)}\right)>\operatorname{card}\left(V_{\alpha(4)}\right)
$$

Furthermore, by (14),

$$
1 \leqq \operatorname{card}\left(V_{\alpha(i)}\right)<\operatorname{card}\left(E_{2}\right)=a, \text { for } i=2,3,4 .
$$

Let $V_{\alpha(1)}=\left\{v_{\alpha(1)}^{1}, \ldots, v_{\alpha(1)}^{\beta}\right\}$. Consider the numbers $v_{i}$ in the equations (16). Choose from (5) $\beta$ permutations $q_{i}(x), i=1, \ldots \beta$, such that

$$
q_{i}\left(v_{\alpha(1)}\right)=v_{\alpha(2)}, q_{i}\left(v_{\alpha(2)}\right)=v_{\alpha(1)}^{i}, \quad q_{i}\left(v_{\alpha(3)}\right)=v_{\alpha(3)} .
$$

Then, for all $i, q_{i}\left(v_{\alpha(4)}\right) \in V_{\alpha(4)}$ because, otherwise, we would obtain a permissible set of representatives whose elements are contained in exactly three sets $V_{i}$.

By (17), this implies that, for some $\mu$ and $v \cdot \mu=v$,

$$
q_{\mu}\left(v_{\alpha(4)}\right)=q_{\nu}\left(v_{\alpha(4)}\right)=v_{\alpha(4)}^{*} \in V_{\alpha(4)} .
$$

We have, thus, constructed the following two permissible sets of representatives which differ by one element only

$$
\left(v_{\alpha(2)}, v_{\alpha(1)}^{\mu}, v_{\alpha(3)}, v_{\alpha(4)}^{*}\right) ;\left(v_{\alpha(2)}, v_{\alpha(1)}^{v}, v_{\alpha(3)}, v_{\alpha(4)}^{*}\right) .
$$

We now choose from (5) a permutation $q^{\prime}(x)$ such that

$$
q^{\prime}\left(v_{\alpha(1)}^{\mu}\right)=v_{\alpha(2)}, q^{\prime}\left(v_{\alpha(1)}^{v}\right)=v_{\alpha(3)}, q^{\prime}\left(v_{\alpha(3)}\right)=v_{\alpha(1)} .
$$

Consider the values

$$
q^{\prime}\left(v_{\alpha(2)}\right) \text { and } q^{\prime}\left(v_{\alpha(4)}^{*}\right)
$$


Because the sets (19) are permissible and $q^{\prime}$ obviously maps a permissible set into a permissible set, the values (20) are both contained in the set $V_{\alpha(1)}$. Otherwise, we would obtain a permissible set of representatives whose elements are contained in exactly three sets $V_{i}$.

We may now choose

$$
g_{1}(x)=\bar{g} q^{\prime} q_{\mu} \bar{g}(x)
$$

The function $g_{1}(x)$ assumes the value $v_{\alpha(2)}$, for $x \in V_{\alpha(2)}$, and the value $v_{\alpha(1)}$, otherwise. By (18), it is of type $\left[n-a_{1}, a_{1}\right]$ where $1 \leqq a_{1}<a$. This completes the proof of lemma 5 .

Proof of the theorem. We assume first that $n \geqq 5, n \neq 2^{r}$ and (5) is a triply transitive group of degree $n$. Let $f\left(x_{1}, \ldots, x_{k}\right)$ be an arbitrary function satisfying Słupecki conditions. To show that $\mathfrak{B S}$ is basic for $\mathfrak{E}_{n}$, we prove that the set $\mathfrak{F}$ consisting of $\mathscr{S}$ and $f$ is complete.

By lemma 3, $₹$ generates a function $g(x)$ of genus 2. This implies, by lemma 5, that $\mathfrak{F}$ is complete, provided $g(x)$ is not of type

$$
\left[\frac{1}{2} n, \frac{1}{2} n\right] \text {. }
$$

We assume that $g(x)$ is of type (21) and that $E_{1}$ and $E_{2}$ are disjoint subsets of $N$ such that card $\left(E_{1}\right)=$ card $\left(E_{2}\right)=\frac{1}{2} n$ and $g(x)$ assumes a constant value both in $E_{1}$ and in $E_{2}$. We shall now proceed as in the proof of lemma 5 .

We form the sets $V_{i}, i=1,2,3,4$, and obtain a function $\bar{g}(x)$ satisfying the equations (16). (Otherwise, we would obtain a function of genus 2 and not of type (21) which would complete the proof.) Furthermore, we may assume that the sets $V_{i}$ are of the same cardinality because, otherwise, we could use the inequality (17) as in the proof of lemma 5 . Thus, the set $N$

\begin{tabular}{|c|c|c|c|}
\hline \multicolumn{4}{|c|}{$N$} \\
\hline \multicolumn{2}{|c|}{$E_{1}$} & \multicolumn{2}{|c|}{$E_{2}$} \\
\hline$V_{1}$ & $V_{4}$ & $V_{2}$ & $V_{3}$ \\
\hline
\end{tabular}
is divided into subsets as follows:

We now form a new partition of $N$ into $V$-sets by choosing from of a permutation $\bar{p}(x)$ which maps some element of $V_{1}$ into itself and some other element of $V_{1}$ into $V_{3}$ and denoting

$$
V_{1}^{1}=E_{1} \cap \bar{p}\left(E_{1}\right), V_{2}^{1}=E_{2} \cap \bar{p}\left(E_{1}\right), V_{3}^{1}=E_{2} \cap \bar{p}\left(E_{2}\right), V_{4}^{1}=E_{1} \cap \bar{p}\left(E_{2}\right) .
$$

Again, we may conclude that the sets $V_{i}^{1}$ are of the same cardinality. Furthermore, we may assume that the following equations hold: 


$$
\begin{aligned}
\operatorname{card}\left(V_{1} \cap V_{1}^{1}\right) & =\operatorname{card}\left(V_{1} \cap V_{4}^{1}\right)=\operatorname{card}\left(V_{4} \cap V_{1}^{1}\right)=\operatorname{card}\left(V_{4} \cap V_{4}^{1}\right) \\
& =\operatorname{card}\left(V_{2} \cap V_{2}^{1}\right)=\operatorname{card}\left(V_{2} \cap V_{3}^{1}\right)=\operatorname{card}\left(V_{3} \cap V_{2}^{1}\right) \\
& =\operatorname{card}\left(V_{3} \cap V_{3}^{1}\right)=\frac{1}{2} \operatorname{card}\left(V_{1}\right)=\frac{1}{4} \operatorname{card}\left(E_{1}\right) \\
& =\frac{1}{8} \operatorname{card}(N)=\frac{1}{8} n .
\end{aligned}
$$

For if the equations (22) do not hold we may argue as follows. Assume that, for instance,

$$
\operatorname{card}\left(V_{1} \cap V_{1}^{1}\right)>\operatorname{card}\left(V_{1} \cap V_{4}^{1}\right) .
$$

Let $V_{1} \cap V_{1}^{1}=\left\{\bar{v}_{1}, \ldots, \bar{v}_{\gamma}\right\}$. We choose from (5) permutations $\pi_{i}(x)$, $i=1, \ldots, \gamma$, such that $\pi_{i}\left(v_{1}\right)=\bar{v}_{i}, \pi_{i}\left(v_{2}\right)$ equals some fixed element in $V_{4} \cap V_{1}^{1}$ and $\pi_{i}\left(v_{3}\right)$ equals some fixed element in $V_{4} \cap V_{4}^{1}$. If, for some $i$, $\pi_{i}\left(v_{4}\right) \notin V_{1} \cap V_{4}^{1}$ we obtain a function of genus 2 and not of type (21). If, for all $i, \pi_{i}\left(v_{4}\right) \in V_{1} \cap V_{4}^{1}$ we obtain, by (23), two permissible sets of

\begin{tabular}{|c|c|c|c|c|c|c|c|}
\hline \multicolumn{8}{|c|}{$N$} \\
\hline \multicolumn{4}{|c|}{$E_{1}$} & \multicolumn{4}{|c|}{$E_{2}$} \\
\hline \multicolumn{2}{|c|}{$V_{1}$} & \multicolumn{2}{|c|}{$V_{4}$} & \multicolumn{2}{|c|}{$V_{2}$} & \multicolumn{2}{|c|}{$V_{3}$} \\
\hline$V_{1}^{1}$ & $V_{4}^{1}$ & $V_{1}^{1}$ & $V_{4}^{1}$ & $V_{2}^{1}$ & $V_{3}^{1}$ & $V_{2}^{1}$ & $V_{3}^{1}$ \\
\hline
\end{tabular}
representatives differing by one element only. Then we may argue as in the proof of lemma 5 .

Equations (22) express the fact that $N$ is divided into subsets as follows:

We continue the process by forming a new partition of $N$ into sets $V_{i}^{2}$, $i=1,2,3,4$. If we do not obtain a function of genus 2 and of some type other than $(21)$ we obtain equations corresponding to (22). The common cardinality of the sets involved equals $\frac{1}{16} n$.

By repeating the argument for new partitions of $S$, we conclude that we either obtain a function of genus 2 and not of type $(21)$ or $n=2$. Thus, the part of our theorem concerning triply transitive groups follows.

Assume that $n \geqq 5$ and (5) is a quadruply transitive group of degree $n$. Let $\mathfrak{F}$ be as above. The completeness of $\mathfrak{F}$ follows because we may choose from (S) a permutation mapping the numbers $v_{i}, i=1,2,3,4$, into exactly three of the sets $V_{i}$. We, thus, obtain a permissible set of representatives whose elements are contained in exactly three sets $V_{i}$.

Therefore, we have established our theorem. We note, finally, that the main difficulties in the proof are due to the fact that no analogues of lemma 1.2 in [3] are available. 
3. Special cases. We shall now show that the condition $n \neq 2^{r}$ in the statement of our theorem is essential. If $n=2^{r}(r \geqq 2)$ there is a triply transitive group of degree $n$ which is not a basic group for $\mathfrak{E}_{n}$. In what follows, we shall discuss the case $n=8$ in detail.

Let $\mathbb{S S}_{8}$ be the holomorph of an Abelian group of order 8 and type $(1,1,1)$, expressed in the usual way as a permutation group of degree 8 . $\mathbb{B S}_{8}$ is generated by the two permutations (1376528) and (17)(46). It is of order 1344 and consists of 3847 -cycles, 224 permutations of cyclic structure $3 \times 3,224$ permutations of cyclic structure $6 \times 2,252$ permutations of cyclic structure $4 \times 4,49$ permutations of cyclic structure $2 \times 2 \times 2 \times 2,42$ permutations of cyclic structure $2 \times 2,168$ permutations of cyclic structure $4 \times 2$ and the identity. The group $\mathscr{G}_{8}$ can also be characterized by the following six defining relations:

$$
\begin{gathered}
X^{7}=1, Y^{2}=1,\left(Y X^{3}\right)^{4}=1,(Y X)^{6}=1 \\
\left(Y X^{3} Y X^{2} Y X\right)^{2}=1, Y X^{3}(Y X)^{2} Y X^{4} Y X^{5} Y X^{6} Y X^{5}=1 .
\end{gathered}
$$

Obviously, the holomorph of an Abelian group of order $2^{r}$ and type $(1,1, \ldots, 1)$ (i.e. the holomorph of a so-called generalized Klein group) is triply transitive. In particular, $\mathscr{G S}_{8}$ is triply transitive.

However, $\mathfrak{B S}_{8}$ is not a basic group for $\mathfrak{F}_{8}$. Consider the following function $f(x, y)$ which satisfies Słupecki conditions:

$$
f(2 x-1, y)=y, f(2 x, y)=9-y .
$$

Then the set $\mathfrak{F}$ consisting of $\mathfrak{G F}_{8}$ and $f(x, y)$ is not complete.

To prove this, we quote some terminology and notations, from section 2 . We let $\quad E_{1}=\{1,2,3,4\}, \quad E_{2}=\{5,6,7,8\}, \quad V_{1}=\{1,2\}, \quad V_{4}=\{3,4\}$, $V_{2}=\{5,6\}$ and $V_{3}=\{7,8\}$. The following (unordered) quadruples are called permissible sets of representatives:

$$
\begin{array}{lllllll}
1234, & 1256, & 1278, & 1357, & 1368, & 1458, & 1467, \\
2358, & 2367, & 2457, & 2468, & 3456, & 3478, & 5678 .
\end{array}
$$

The permutations in $\mathbb{S}_{8}$ always map a permissible set of representatives into a permissible set. Furthermore, they preserve the subset structure (24) of $N$.

Let $\mathfrak{F}_{8} \subset \mathfrak{F}_{8}$ be the set consisting of the following 1-place functions:

1) Permutations in $\mathfrak{S Y}_{8}$.

2) Constants.

3) Those functions of type $[2,2,2,2]$ whose values form a permissible set of representatives and which, furthermore, assume a constant value in the sets $V_{1}^{i}, V_{2}^{i}, V_{3}^{i}$ and $V_{4}^{i}$, for some $i=1, \ldots, 7$, where 


$$
\begin{aligned}
& V_{1}^{1}=\{1,2\}, \quad V_{2}^{1}=\{3,4\}, \quad V_{3}^{1}=\{5,6\}, \quad V_{4}^{1}=\{7,8\} ; \\
& V_{1}^{2}=\{1,3\}, \quad V_{2}^{2}=\{2,4\}, \quad V_{3}^{2}=\{5,7\}, \quad V_{4}^{2}=\{6,8\} ; \\
& V_{1}^{3}=\{1,4\}, \quad V_{2}^{3}=\{2,3\}, \quad V_{3}^{3}=\{5,8\}, \quad V_{4}^{3}=\{6,7\} ; \\
& V_{1}^{4}=\{1,5\}, \quad V_{2}^{4}=\{2,6\}, \quad V_{3}^{4}=\{3,7\}, \quad V_{4}^{4}=\{4,8\} ； \\
& V_{1}^{5}=\{1,6\}, \quad V_{2}^{5}=\{4,7\}, \quad V_{3}^{5}=\{2,5\}, \quad V_{4}^{5}=\{3,8\} ; \\
& V_{1}^{6}=\{1,7\}, \quad V_{2}^{6}=\{3,5\}, \quad V_{3}^{6}=\{2,8\}, \quad V_{4}^{6}=\{4,6\} ; \\
& V_{1}^{7}=\{1,8\}, \quad V_{2}^{7}=\{4,5\}, \quad V_{3}^{7}=\{2,7\}, \quad V_{4}^{7}=\{3,6\} .
\end{aligned}
$$

4) Those functions of type $[4,4]$ which, for some $i$, assume a constant value in one of the sets $V_{1}^{i} \cup V_{2}^{i}, \quad V_{1}^{i} \cup V_{3}^{i}$ or $V_{1}^{i} \cup V_{4}^{i}$.

The set $\mathfrak{F}_{8}$ is closed under composition. In classes 1)-4) there are, respectively, 1344, 8, 2352 and 392 functions. Thus, card $\left(\widetilde{F}_{8}\right)=4096$. This number can be computed more directly as follows. $\tilde{\mho}_{8}$ consists of all functions which map every permissible set of representatives into a permissible set, a quadruple of type $[2,2]$ or of type [4]. (In what follows, quadruples of these three forms are called permissible images.) Thus, we may choose arbitrarily the values $h(1), h(2), h(3)$ of a function $h(x) \in \mathfrak{F}_{8}$. They determine uniquely the value $h(4)$. Again, $h(5)$ may be chosen arbitrarily but then the values $h(6), h(7), h(8)$ are uniquely determined. Hence,

$$
\operatorname{card}\left(\mathfrak{F}_{8}\right)=8^{4}=4096 \text {. }
$$

Our function $f(x, y)$ forms a closure in the set $\mathfrak{F}_{8}$, i.e. if $g_{1}(x), g_{2}(x) \in \widetilde{\mho}_{8}$ then also $f\left(g_{1}(x), g_{2}(x)\right) \in \mathfrak{F}_{8}$. To prove this, it suffices to show that if $\left(i_{1}, i_{2}, i_{3}, i_{4}\right)$ and $\left(j_{1}, j_{2}, j_{3}, j_{4}\right)$ are two permissible images then also

$$
\left(f\left(i_{1}, j_{1}\right), f\left(i_{2}, j_{2}\right), f\left(i_{3}, j_{3}\right), f\left(i_{4}, j_{4}\right)\right)
$$

is a permissible image. This can be readily verified by considering the matrix of $f(x, y)$.

Thus, $\mathfrak{F}$ generates no 1-place functions other than the functions in $\widetilde{F}_{8}$. This proves that $\mathfrak{F}$ is not complete. Clearly, instead of the function $f(x, y)$, we may choose any function which satisfies Słupecki conditions and forms a closure in the set $\widetilde{\mho}_{8}$.

Consider the general case $\left.{ }^{1}\right) n=2^{r}, r \geqq 3$. Let $\mathbb{S}_{2^{r}}$ be the holomorph of an Abelian group of order $2^{r}$ and type $(1,1, \ldots, 1)$. The order of this triply transitive group $\mathscr{G S}_{2^{r}}$ equals

$$
2^{r}\left(2^{r}-1\right)\left(2^{r}-2\right)\left(2^{r}-2^{2}\right) \cdots\left(2^{r}-2^{r-1}\right) .
$$

1) We have regarded the case $n=8$ as the first exceptional case. In fact, also the case $n=4$ may be considered as exceptional, the exceptional group being the holomorph of the four-group (which equals the symmetric group of degree 4). Our theorem is not valid for $n=3$ because lemma 3 is not valid in this case. 
Define a function $\varphi(x, y) \in \mathcal{E}_{2^{r}}$ as follows:

$$
\varphi(2 x-1, y)=y, \quad \varphi(2 x, y)=2^{r}+1-y .
$$

The function $\varphi(x, y)$ forms a closure in a set $\mathfrak{F}_{2^{r}}$ consisting of $2^{r(r+1)}$ 1-place functions. This implies that the set $\mathfrak{F}$ consisting of $\mathscr{G}_{2}{ }^{r}$ and $\varphi(x, y)$ is not complete. Hence, the group $\mathscr{G}_{2^{r}}$ is not a basic group for $\mathfrak{F}_{2^{r}}$.

\section{References}

[1] Яьлонский, С. В.: Функциональные построения в $k$-значной гогике. - Тр. Матем. инст. им. В. А. Стеклова, 51 (1958), 5-142.

[2] SalomaA, A.: A theorem concerning the composition of functions of several variables ranging over a finite set. - J. Symbolic Logic 25 (1960), 203-208.

[3] - - Some completeness criteria for sets of functions over a finite domain. I. Ann. Univ. Turkuensis, Ser. A I 53 (1962). 